\title{
FALÊNCIA HEPÁTICA FULMINANTE: ETIOLOGIA, MANEJO E INDICAÇÃO PARA O TRANSPLANTE DE FÍGADO
}

\author{
Fulminant hepatic failure: etiology, management and indications for liver trans- \\ plantation
}

\author{
Olival Cirilo Lucena da FONSECA-NETO
}

\begin{abstract}
Fonseca-Neto OCL. Falência hepática fulminante: etiologia, manejo e indicação para o transplante de fígado. ABCD Arq Bras Cir Dig 2008;21(4): 201-4 RESUMO - Introdução - Falência hepática fulminante (FHF) é síndrome que se caracteriza pelo desenvolvimento de dano hepático com diminuição da capacidade sintética e encefalopatia em pacientes com função hepática normal prévia ou pelo menos com doença hepática compensada oculta. É uma das mais desafiantes emergências gastrointestinais encontradas na prática clínica e envolve padrão de sintomas clínicos e respostas fisiopatológicas associadas com a rápida parada da função hepática normal. Métodos - Realizou-se revisão bibliográfica de artigos científicos publicados e disponíveis no Pub-Med, através do site www.pubmed.com cruzando-se os descritores: falência hepática fulminante, transplante de fígado, hepatite. FHF permanece síndrome potencialmente devastadora com alta taxa de mortalidade. O bom manejo é multidisciplinar e mais do que $50 \%$ dos pacientes recebem transplante de fígado, com sobrevida em torno de 75-90\%. Conclusão - Apesar das opções terapêuticas especificas nas causas distintas, o transplante ortotópico de fígado é a única terapêutica que proporciona melhora da sobrevida na maioria dos pacientes. A utilização dos sistemas hepáticos artificiais em pacientes com FHF até o momento ainda não recompensa.

DESCRITORES - Falência hepática aguda. Transplante de fígado. Hepatite.
\end{abstract}

\section{INTRODUÇÃO}

Falência hepática fulminante (FHF) é síndrome complexa que reflete rápida progressão, risco de vida potencialmente reversível e deterioração da função hepática em pacientes sem doença prévia no fígado ${ }^{23}$. Desde o início de 1990 ocorreu mudança na epidemiologia da FHF. A origem viral vem diminuindo nos países ocidentais e a injúria induzida por drogas, acetaminofeno, vem assumindo a liderança. Nos últimos 20 anos, transplante de fígado de emergência emergiu como a única intervenção terapêutica que beneficia os pacientes com FHF avançada ${ }^{22}$.

\section{ETIOLOGIA}

As causas da FHF variam grandemente entre os países. Nos EUA, em 1960, as mais frequentes eram a hepatite A e B28. No momento atual, a hepatite B constitui apenas $7 \%$, enquanto o acetominofeno atinge próximo de $50 \%$ de todos os $\operatorname{casos}^{17}$. Igualmente os países em desenvolvimento apresentam quadro diferente quando comparados com a

Trabalho realizado no Serviço de Cirurgia Geral e Transplante Hepático do Hospital Universitário Oswaldo Cruz, Recife, PE, Brasil.

Correspondência: Olival Cirilo Lucena Fonseca Neto, e-mail: olivalneto@globo.com
América do Norte e Europa. A determinação etiológica permanece muito importante, pois orienta como tratar e qual é o prognóstico esperado ${ }^{16}$.

A apresentação clínica vai depender da severidade e da etiologia do dano hepático. Sintomas inespecíficos como náuseas, vômitos e desconforto abdominal poderão ser apresentação inicial dos pacientes com $\mathrm{FHF}^{20}$. A evolução desses sintomas ou o aparecimento desde o início de confusão mental, sangramento no trato digestório e hipoglicemia tornarão o diagnóstico mais óbvio ${ }^{6}$. Os resultados dos testes de função hepática e coagulação indicarão a severidade da agressão no fígado ${ }^{5}$. Praticamente, todos os sistemas são acometidos direta ou indiretamente pela disfunção grave hepática ${ }^{4}$. Ela pode apresentar encefalopatia, coagulopatia, alteração cardiovascular e nefrometabólica.

A encefalopatia ${ }^{15}$ pode variar desde a apresentação subclínica e dificuldades na concentração (estágio I) ao coma profundo (estágio IV). O seu aparecimento e evolução para os estágios mais graves (grau IV) estão inversamente relacionados com o prognóstico, ou seja, maior estágio da encefalopatia pior prognóstico. Edema cerebral é distúrbio neurológico comum na FHF, sendo reportado na vasta maioria dos casos que progridem para o estágio IV da encefalopatia e é a principal causa de morte nos pacientes com FHF.

O fígado exerce papel fundamental na síntese da 
maioria dos fatores de coagulação ${ }^{1}$ e de alguns inibidores da coagulação e fibrinólise. Disfunção plaquetária (defeito quantitativo e qualitativo), redução dos níveis séricos de fibrinogênio, fatores II, V, VII, IX e X e déficit dos inibidores da coagulação (antitrombina III, proteína $\mathrm{C}$ e $\mathrm{S}$ ) podem produzir grande hemorragia. O tempo de protrombina (INR) é usado como indicador de severidade da injúria hepática.

Vasodilatação sistêmica, resistência vascular sistêmica diminuída, hipotensão e aumento do débito cardíaco caracterizam o status hemodinâmico na FHF e são as suas principais alterações cardiovasculares ${ }^{10}$. Hipóxia e acidose láctica poderão aparecer com a progressão das disfunções orgânicas.

As alterações nefro-metabólicas principais consistem em falência renal aguda (hepatorenal ou por hipovolemia), anormalidades eletrolíticas, hipoglicemia e pancreatite.

\section{MANEJO CLÍNICO}

Os componentes para melhor estratégia no manejo dos pacientes com FHF são: determinar a causa, avaliar a severidade da doença e associar o prognóstico, prevenir ou tratar as disfunções orgânicas, realizar transplante de figado quando a recuperação espontânea é improvável, e, se possível, utilizar suporte hepático artificial ${ }^{2,9}$.

A deterioração clínica rápida, o aparecimento de falências orgânicas graves e a necessidade de tomadas de decisões urgentes configuram desafio clínico diante dos pacientes com $\mathrm{FHF}^{12}$.

Na monitorização das funções orgânicas faz-se necessário o uso de cateter venoso central (pressão venosa central e saturação venosa central); cateter vesical (diurese e pressão intraabdominal); cateter arterial periférico (pressão arterial média, gasometria e lactato); cateter de artéria pulmonar (eventual) e cateter para medir pressão intra-craniana (eventual).

A preocupação com a infecção deve estar presente já que nesses pacientes a barreira intestino-fígado está danificada colocando diretamente na corrente sanguínea toxinas esplâncnicas ou mesmo microorganismos (translocação bacteriana). Além disso, a necessidade da monitorização invasiva permite a quebra das barreiras de proteção cutânea ${ }^{14}$.

$\mathrm{O}$ transplante de fígado revolucionou o tratamento dos pacientes com $\mathrm{FHF}^{7}$. Antes da era do transplante, apenas 1/5 dos pacientes acometidos de falência hepática aguda sobreviviam. Atualmente, com os refinamentos na técnica cirúrgica, melhores cuidados no manejo intensivo e introdução de drogas imunossupressoras mais seletivas ele oferece sobrevida em torno de $65 \%$ e, em algumas séries, chegando a mais de $80 \%^{3}$.

Apesar de todo este esforço da comunidade transplantadora, os pacientes portadores de FHF apresentam pior evolução do que os com doença hepática crônica transplantados.

Todos os pacientes que apresentam os critérios de indicação de transplante devem ser listados para receberem um novo órgão.

Devido à escassez de órgãos para transplante muitos dos pacientes com FHF desenvolvem falências orgânicas graves e, com isso, contraindicação ao transplante hepático ${ }^{8}$.

O transplante hepático com doador vivo aparentado é hoje nova modalidade bem estabelecida no transplante eletivo. Devido à falta de órgãos de doadores falecidos, essa opção também vem sendo utilizada nos portadores de FHF com bons resultados ${ }^{18}$.

O transplante de fígado auxiliar é técnica em que um órgão parcial é colocado de maneira heterotópica (sem retirar o figado nativo, o órgão novo parcial é colocado em algum local na cavidade abdominal, por exemplo, na loja esplênica após a retirada do baço) ou ortotópica (uma parte do fígado nativo é retirado e o fígado parcial novo é colocado no lugar) enquanto o próprio fígado "regenera"13. É como se desse "suporte hepático cirúrgico" garantindo a vida do paciente caso isso não aconteça. Se ele retornar às funções hepáticas normais, o figado parcial transplantado pode ser removido ou deixá-lo atrofiar.

A hepatectomia total com shunt porto-cava foi realizada, e em algumas séries apresentou evolução favorável. O racional seria a retirada do foco tóxico liberador das citocinas inflamatórias (o fígado) e, com isso, levaria à maior estabilidade hemodinâmica até a oferta do novo órgão. Claro que é um procedimento heróico e controverso ${ }^{19}$.

O transplante de hepatócitos representa alternativa biológica atraente que permitiria aumento na função hepática. Ainda é preciso estudos bem controlados para comprovar seu benefício. Com o avanço da genética, a utilização de xenoenxertos (fígado de porco) tem sido proposta, porém a rejeição entre as espécies aparece como barreira a ser quebrada ${ }^{26}$.

Conhecendo-se a propriedade do fígado em "regenerar", admite-se que os pacientes com FHF que se recuperam, tendem a recompor a anatomia funcional hepática ${ }^{24}$. Assim, o tratamento ideal seria manter artificialmente a função hepática até que o figado pudesse recompor as suas funções. Várias foram as tentativas no passado para realizar esse objetivo: circulação extracorpórea com fígado heterólogo e, depois hemoperfusão em carvão ativado. Evidente que lograram sucesso. Nos últimos anos, a utilização de sistemas bioartificiais com hepatócitos humanos ou de outras espécies vem sendo utilizados com algum sucesso nos pacientes com $\mathrm{FHF}^{27}$.

Entretanto, a utilização de sistemas extra-corpóreos artificiais, como diálise com albumina e o sistema de membranas MARS (molécula absorbing recirculating system) vem surgindo como princípio terapêutico promissor ${ }^{21}$. O MARS é suporte de fígado artificial. Ele tem potencial de eliminar as substâncias ligadas à albumina, bilirrubina, amônia e aminoácidos aromáticos. O racional para seu uso é dar suporte para possível melhora do quadro clínico e, aí então, avaliar a real necessidade do transplante de fígado ou levar o paciente para o transplante hepático em melhores condições aumentando as chances de sucesso do procedimento. $\mathrm{O}$ verdadeiro valor dessas terapêuticas deverá ser estabelecido ${ }^{30}$.

O prognóstico da FHF varia de acordo com a causa ${ }^{25}$. Predizer a evolução dos pacientes com FHF é importante devido à necessidade do manejo efetivo e à possibilidade da transferência para centros com programa de transplante de fígado.

Vários critérios de prognóstico são utilizados em todo o mundo. No entanto, os mais utilizados são "King's College Criteria" e "Clichy Criteria" (Tabela 1). Vale lembrar que os 
pacientes que preenchem esses critérios apresentam baixas chances de sobrevida com o tratamento clínico, assim, existe indicação imediata do transplante ${ }^{11}$.

TABELA 1 - Critérios para indicação de transplante hepático nas FHF

\begin{tabular}{|c|c|c|}
\hline $\begin{array}{l}\text { Clichy - Paul } \\
\text { Brousse }\end{array}$ & $\begin{array}{l}\text { Fator } \mathrm{V}<30 \%, \text { se }>30 \text { anos } \\
\text { Fator } \mathrm{V}<20 \% \text {, se }<30 \text { anos } \\
\text { Encefalopatia grau } 3 \text { ou } 4\end{array}$ & \\
\hline \multirow[t]{3}{*}{ King's College } & Paracetamol & Não - Paracetamol \\
\hline & $\begin{array}{l}\text { - sozinho, } \mathrm{ph}<7,3 \\
\text { - } \mathrm{TP}>100 \mathrm{~s}+\mathrm{Cr}>3,4 \mathrm{mg} \% \\
\text { + encefalopatia grau } 3 \text { ou } 4\end{array}$ & $\begin{array}{l}\text { - TP }>100 \text { s (sozinho, já indica) } \\
\text { ou } 3 \text { dos seguintes critérios: } \\
\text { etiologia desfavorável (hepatite } \\
\text { não-A, não-B, halotano, subful- } \\
\text { minante*). }\end{array}$ \\
\hline & & $\begin{array}{l}-\mathrm{TP}>50 \mathrm{~s}, \text { bilirrubina }>17,5 \\
\mathrm{mg} \%\end{array}$ \\
\hline
\end{tabular}

Mais recentemente a utilização do lactato sérico $(\geq 30)$ após correção da hipovolemia e a identificação de hiperfosfatemia sérica $(\geq 3)$ - que mostram a falência renal e insuficiente regeneração hepática -, permitiu identificação mais rápida e prática da gravidade desses pacientes. No entanto, aguarda-se validação desses achados em estudos mais apropriados.

O MELD (model for end-stage liver disease), escore matemático utilizado para alocação de enxertos hepáticos nos EUA e aqui no Brasil também vem sendo utilizado para identificar precocemente os candidatos ao transplante hepático ${ }^{29}$.

Apesar das muitas opções encontradas na literatura para predizer a evolução dos pacientes com falência hepática aguda, nenhuma suplanta o bom julgamento clínico.

\section{CONCLUSÃO}

A falência hepática aguda é doença devastadora com um número indefinido de causas. O diagnóstico prenuncia evolução quase letal relacionada com deterioração neurológica, sepse, disfunção de múltiplos órgãos e múltiplos efeitos associados ao não-funcionamento do fígado. O reconhecimento precoce da doença e transferência imediata para um centro transplantador é fundamental para abordagem multidisciplinar e monitorização intensiva. Apesar do transplante de fígado ser a única opção efetiva, para aqueles que não se recuperam clinicamente a mortalidade permanece alta devido à falta de órgãos para transplante que limita a indicação de seu uso nesta situação.

Fonseca-Neto OCL. Fulminant hepatic failure: etiology, management and indications for liver transplantation. ABCD Arq Bras Cir Dig 2008;21(4):201-4 ABSTRACT - Background - Fulminant hepatic failure (FHF) is characterized by the development of liver injury with decrease synthetic capacity and encephalopathy in patients with previous normal liver or, at least, well compensated liver disease. It is one of the most challenging gastrointestinal emergencies encountered in clinical practice and encompasses a pattern of clinical symptoms and pathophysiological responses associated with the rapid arrest of normal hepatic function. Methods - A literature review was made in articles available at Pub Med site (www.pubmed.com), crossing the following headings: fulminant hepatic failure, liver transplantation e hepatitis. FHF remains a potentially devasting syndrome with a high mortality rate. The optimal care is multidisciplinary and up to half of the cases receive liver transplants, with survival rates around $75-90 \%$. Conclusions - In spite of specific therapeutic options in distinctive etiologies, orthotopic liver transplant is the only therapy proven to improve patient survival in the majority of patients. The search for artificial liver support systems to manage patients with FHF has been unrewarding fully to date.

HEADINGS - Liver failure, acute. Liver transplantation. Hepatitis.

\section{REFERÊNCIAS}

1. Auzinger G, Wendon J. Intensive care management of acute liver failure. Current Opinion in Critical Care 2008; 14:179-188.

2. Bernal W, Auzinger G, Sizer E, Wendon J. Intensive care management of acute liver failure. Seminars in Liver Disease 2008; 28:188-200.

3. Bernal W, Wendon J. Liver transplantation in adults with acute liver failure. J Hepatol 2004; 40:192-197.

4. Blei AT. Medical therapy of brain edema in fulminant hepatic failure. Hepatology 2000; 32(3):666-669.

5. Brandsater B, Höckerstedt K, Friman S, Ericzon BG, Kirkegaard P, Isoniemi H, Olausson M, Broome U, Schmidt L, Foss A, Bjoro K. Fulminant hepatic failure: outcome after listing for highly urgent liver transplantation - 12 years experience in the Nordic Countries. Liver Transplantation 2002; 8(11):10551062.

6. Caraceni P, Thiel DHV. Acute liver failure. The Lancet 1995; 345:163-69.

7. Castaldo ET, Chari RS. Liver transplantation for acute hepatic failure. HPB 2006; 8:29-34

8. Castells A, Salmerón JM, Navasa M, Rimola A, Salo J, Andreu H, Mas A, Rodés J. Liver transplantation for acute liver failure: analysis of applicability. Gastroenterology 1993; 105:532-538.

9. Clavien PA. Acute liver failure: where are the challenges? Journal of Hepatology 2007 ; 46:553-582.

10. Detry $\mathrm{O}$, Honoré $\mathrm{P}$, Meurisse $\mathrm{M}$, Jacquet N. Management of fulminant hepatic failure. Acta Chir Belg 1998; 98:235-240.

11. Dhiman RK, Jain S, Maeshwari U, et al. Early indicators of prognosis in fulminant hepatic failure: an assessment of the model for end-stage liver diasease (MELD) and King's College Hospital criteria. Liver Transpl 2007; 13:814-821.
12. Han MK, Hyzy R. Advances in critical care management of hepatic failure and insufficiency. Crit Care Med 2006; 34(9)S225-S231.

13. Jaeck D, Pessaux P, Wolf $P$. Which types of graft to use in patients with acute liver failure? (A) Auxiliary liver transplant (B) Living donor liver transplantation (C) The whole liver (A) I prefer auxiliary liver transplant. Journal of Hepatology 2007; 46:553-582.

14. Larsen FS, Strauss G, Knudsen GM, Herzog TM, Hansen BA, Secher NH. Cerebral perfusion, cardiac output, and arterial pressure in patients with fulminant hepatic failure. Crit Care Med 2000; 28(4):996-1000.

15. Larsen FS, Wendon J. Brain edema in liver failure: basic physiologic principles and management. Liver Transplantation 2002; 8(11):983-989.

16. Lee WM, Seremba E. Etiologies of acute liver failure. Current Opinion in Critical Care 2008; 14:198-201.

17. Lee WM. Etiologies of acute liver failure. Semin Liver Dis 2008; 28:142-152.

18. Liou IW, Larson AM. Role of liver transplantation in acute liver failure. Seminars in Liver Disease 2008; 28(2):201-209.

19. Lodge JPA, Dasgupta D, Prasad KR, Attia M, Toogood GJ, Davies M, Millson C, Breslin N, Wyatt J, Robinson PJ, Bellamy MC, Snook N, Pollard SG. Emergency subtotal hepatectomy: a new concept for acetaminophen-induced acute liver failure. Ann Surg 2008; 247(2):238-249.

20. Mas A, Rodés J. Fulminant hepatic failure. Lancet 1997; 349:1081-85.

21. McKenzie TJ, Lillegard JB, Nyberg SL. Artificial and bioartificial liver support. Seminars in Liver Disease 2008; 28(2):210-217.

22. Merritt WT. Perioperative concerns in acute liver failure. Int Anesthesiol Clin 2006; 44(4):37-57. 
23. O’Grady JG. Acute liver failure. Postgrad Med J 2005; 81:148-154.

24. Phua J, Lee KH. Liver support devices. Current Opin Crit Care 2008; 14:215

25. Polson J. Assessment of prognosis in acute liver failure. Seminars in Liver Disease 2008; 28(2):218-225.

26. Riordan SM, Williams R. Perspectives on liver failure: past and future. Seminars in liver disease 2008; 28(2):137-141.

27. Rutherford A, Chung RT. Acute liver failure: mechanisms of hepatocyte injury and regeneration. Seminars in Liver Disease 2008; 28(2):167-174.

28. Sass DA, Shakil AO. Fulminant hepatic failure. Liver Transplantation 2005; 11(6):594-605.
29. Schmidt LE, Larsen FS. Prognostic implications of hyperlactatemia, multiple organ failure, and systemic inflammatory response syndrome in patients with acetaminophen induced acute liver failure. Crit Care Med 2006; 34:337-343.

30. Williams R. Correction of disturbed pathophysiology of hepatic failure by albumin dialysis. Hepatobiliary Pancreat Dis Int 2008; 7:19-24.

Fonte de financiamento: não há Conflito de interesse: não há Recebido para publicação: 25/05/2008 Aceito para publicação: 17/08/2008 